\title{
Characterization of small-molecule inhibitors of the sodium iodide symporter
}

\author{
Sabine Lindenthal ${ }^{1}$, Nathalie Lecat-Guillet ${ }^{2}$, Alejandro Ondo-Mendez ${ }^{1,2,3}$, Yves Ambroise ${ }^{2}$, \\ Bernard Rousseau ${ }^{2}$ and Thierry Pourcher ${ }^{1}$ \\ ${ }^{1}$ TIRO, CEA DSV-iBEB-SBTN, CAL, School of Medicine, University of Nice Sophia Antipolis, 28, Avenue de Valombrose, 06107 Nice, France \\ ${ }^{2}$ CEA Saclay, DSV-iBiTecS-SCBM, 91191 Gif-sur-Yvette, France \\ ${ }^{3}$ Laboratory of Biophysics, CIF, University of Colombia, 11001000 Bogota, Colombia \\ (Correspondence should be addressed to T Pourcher; Email: pourcher@unice.fr)
}

\begin{abstract}
The sodium/iodide symporter (NIS) mediates the active transport of iodide from the bloodstream into thyrocytes. NIS function is strategic for the diagnosis and treatment of various thyroid diseases. In addition, a promising anti-cancer strategy based on targeted NIS gene transfer in non-thyroidal cells is currently developed. However, only little information is available concerning the molecular mechanism of NISmediated iodide translocation. Ten small molecules have recently been identified using a high-throughput screening method for their inhibitory effect on iodide uptake of NISexpressing mammalian cells. In the present study, we analyzed these compounds for their rapid and reversible effects on the iodide-induced current in NIS-expressing Xenopus oocytes. Four molecules almost completely inhibited the iodideinduced current; for three of them the effect was irreversible,
\end{abstract}

for one compound the initial current could be fully re-established after washout. Three molecules showed a rapid inhibitory effect of about $75 \%$, half of which was reversible. Another three compounds inhibited the iodideinduced current from 10 to $50 \%$. Some molecules altered the membrane conductance by themselves, i.e. in the absence of iodide. For one of these molecules the observed effect was also found in water-injected oocytes whereas for some others the iodide-independent effect was associated with NIS expression. The tested molecules show a surprisingly high variability in their possible mode of action, and thus are promising tools for further functional characterization of NIS on a molecular level, and they could be useful for medical applications.

Journal of Endocrinology (2009) 200, 357-365

\section{Introduction}

The synthesis of the iodide-containing thyroid hormones requires the active transport and accumulation of iodide from the blood into the thyroid gland that is mediated by the sodium/iodide symporter (NIS). The rat NIS sequence was the first to be identified (Dai et al. 1996), followed by the human (Smanik et al. 1996) and mouse sequences (Perron et al. 2001). The NIS is a glycoprotein residing in the basolateral membrane of thyrocytes (Dohan \& Carrasco 2003). NIS catalyzes the cotransport of one iodide ion together with two sodium ions. For this process, the inwardly directed favourable transmembrane sodium gradient that is maintained by the $\mathrm{Na}^{+} / \mathrm{K}^{+}$-ATPase provides the driving force necessary for the transport of iodide against its electrochemical gradient. Thus, iodide is concentrated in the thyrocytes by up to 40 -fold with respect to the plasma. Iodide is then translocated across the apical membrane of the thyrocytes into the colloid where it is immediately oxidized and organified into thyroglobulin by the thyroid-specific enzyme thyroid peroxidase. The coupling of two iodinated tyrosyl residues within the same thyroglobulin leads to the formation of the precursor molecules of the thyroid hormones T3 and T4. These iodinated thyroglobulins are stored in the colloid until phagocytosis is induced by TSH or pituitary thyrotropin. After hydrolysis, T3 and T4 hormones are released on the basolateral side of the thyrocytes into the bloodstream.

NIS expression and sodium-dependent active iodide transport has also been found in gastric mucosa, salivary gland and lactating mammary gland (Cho et al. 2000, Tazebay et al. 2000). Similar to the thyroid, NIS function generates an important iodide uptake in these tissues. The presence of NIS but no active iodide accumulation was shown in brain, intestine, skin, testis and several other tissues (Perron et al. 2001, Dohan et al. 2003).

NIS also transports various other monovalent anions including $\mathrm{ClO}_{4}^{-}, \mathrm{ClO}_{3}^{-}, \mathrm{SCN}^{-}$and $\mathrm{NO}_{3}^{-}$(Eskandari et al. 1997) and different radioisotopes such as pertechnetate $\left(\mathrm{TCO}_{4}^{-}\right)(\mathrm{Cho}$ et al. 2000, Tazebay et al. 2000, Van Sande et al. 2003), perrhenate $\left(\mathrm{ReO}_{4}^{-}\right)$(Dadachova et al. 2002) and astatine ( $\mathrm{At}^{-}$; Lindencrona et al. 2001). Perchlorate $\left(\mathrm{ClO}_{4}^{-}\right)$is 
the only known efficient competitive inhibitor of NIS activity. Due to the lack of currents in electrophysiological experiments it remained uncertain until recently whether perchlorate is a transported substrate or inhibits the NIS activity (Eskandari et al. 1997, Van Sande et al. 2003, Clewell et al. 2004). Tracer uptake measurements by Van Sande $e t$ al. (2003) with pertechnetate and perrhenate (two large anions that have a similar chemical structure as perchlorate) strongly suggested that perchlorate is translocated into the thyroid via electroneutral transport. Dohan et al. (2007) recently showed that $\mathrm{ClO}_{4}^{-}$is actively transported by NIS in mammary gland of lactating rats leading to its accumulation in the milk. Thus, the stoichiometry of the NIS-mediated sodium/anion symport depends on the translocated anion varying from $2: 1$ for iodide to $1: 1$ or $2: 2$ for perchlorate (Dohan et al. 2007).

Only few organic molecules showing an inhibitory effect on the iodide transport in NIS-expressing tissues have been described so far. Most of these molecules are also known as chloride channel blockers like diphenylamine-2-carboxylate (DPC) and 5-nitro-2-(3-phenylpropylamino)-benzoate (NPPB; Gerard et al. 1994) or as inhibitors of other sodium-dependent transporters like harmaline and a chemical related drug (Kaminsky et al. 1991). Inhibitors of the $\mathrm{Na}^{+} /$ $\mathrm{K}^{+}$-ATPase, like ouabaine, should also lead to the inhibition of the NIS-mediated iodide transport. In this case, no direct interaction with NIS occurs. Inhibition of the $\mathrm{Na}^{+} / \mathrm{K}^{+}$ ATPase abolishes the inward sodium gradient that provides the energy necessary for the iodide translocation. To date, only dysidenin a toxin isolated from the marine sponge Dysidea herbacea has been reported to be a specific NIS inhibitor. Van Sande and collaborators (Van Sande et al. 1990, 2003) found that dysidenin rapidly and strongly inhibits iodide transport in thyroid slices, thyrocyte plasma membrane vesicles and NIS-expressing cell lines (FRTL-5, transfected COS-7). The authors suggest a direct interaction of dysidenin with the NIS iodide binding site.

Specific NIS inhibitors should prove useful for the treatment of various thyroid diseases. For example, amiodarone is a powerful antiarrhythmic and thus, is used in an increasing number of cardiac disorders. But amiodarone is a heavily iodinated drug and its administration induces chronic iodine overload, which frequently causes hyperthyroidism requiring treatment with potassium perchlorate (Bartalena et al. 2004). Due to its only short-lived inhibitory effect frequent, high doses of perchlorate have to be administered causing various undesirable side effects like vomiting, fever and kidney dysfunction. This underlines the need for new NIS inhibitors that could be therapeutic alternatives for perchlorate treatment. NIS inhibitors could also replace perchlorate in the diagnostic of thyroid pathology using ${ }^{123} \mathrm{I}$ scintigraphy and perchlorate depletion tests. They should also prove useful in the case of radioactive iodine contamination (Dayem et al. 2006). A successful strategy in this situation should be a specific inhibition of the NIS-mediated thyroid iodide uptake without simultaneously affecting the secretion and elimination of radioiodine already contained in thyroid hormone precursors. Since to date no specific NIS inhibitor is available, KI consumption is the only protective measure used against thyroid radioiodine accumulation. This treatment is effective mainly when given before or immediately after exposure which considerably reduces its possible application (Dayem et al. 2006).

We here describe an electrophysiological analysis of 10 organic molecules on the iodide-induced current in Xenopus oocytes expressing NIS. These molecules were previously selected (Lecat-Guillet et al. 2008) from a compound collection using a recently developed high-throughput screening method (Lecat-Guillet et al. 2007) for their inhibitory effect on the ${ }^{125} \mathrm{I}^{-}$uptake in permanently NIStransfected HEK-293 cells. These molecules were named ITB-1-ITB-10 for 'Iodide Transport Blocker' (Lecat-Guillet et al. 2008). In the present study, the inhibitory effect of these molecules on the iodide transport was analyzed for its rapidity, reversibility and specificity. The compounds show a high diversity in their possible mode of action.

\section{Materials and Methods}

\section{Material}

Compounds identified as ITB were purchased from a chemical library (Diverset, Chembridge, UK). The purity and identity of each compound were verified by ${ }^{1} \mathrm{H}$ NMR (Bruker avance distrene-plasticizer-xylene 400) and LC-MS (Water, Saint-Quentin en Yvelines, France). Dysidenin and isodysidenin were isolated from the marine sponge $D$. herbacea as reported previously (Lecat-Guillet et al. 2008). The purity of all compounds tested exceeded $98 \%$.

\section{NIS cRNA preparation}

Mouse NIS cRNA was synthesized from the pcDNA3.1 cloning vector (Invitrogen) with the mMessage mMachine T7 Ultra kit (Ambion, Applied Biosystems, Courtaboeuf, France) following the manufacturer's recommendations.

\section{Xenopus laevis oocyte isolation and injection}

Pieces of ovarian lobes were removed from anaesthetized $X$. laevis females (purchased from the 'Centre d'Elevage du CNRS', Montpellier, France) and incubated for $4 \mathrm{~h}$ in $\mathrm{Ca}^{2+}$ free oocyte Ringer solution (Ori) containing $2 \mathrm{mg} / \mathrm{ml}$ collagenase (type A, (Sigma-Aldrich), $1 \mathrm{mg} / \mathrm{ml}$ trypsin inhibitor ((Sigma-Aldrich) and $0 \cdot 1 \mathrm{mg} / \mathrm{ml}$ gentamicin (Invitrogen) at $18{ }^{\circ} \mathrm{C}$.

Stage V-VI oocytes were microinjected (Nanoject II, Drummond, Broomall, PA, USA) with 23 ng NIS cRNA, and the oocytes were kept in Ori at $18^{\circ} \mathrm{C}$ until used for experiments. The composition of Ori was (in $\mathrm{mM}$ ): 110 
$\mathrm{NaCl}, 5 \mathrm{KCl}, 1 \mathrm{MgCl}_{2}, 2 \mathrm{CaCl}_{2}$ and $5 \mathrm{~N}$-2-hydroxyethylpiperazine- $N^{\prime}$-ethanesulphonic acid (HEPES) $(\mathrm{pH} 7 \cdot 5)$.

\section{Electrophysiological measurements}

Conventional two-microelectrode voltage clamp was performed to study $\mathrm{Na}^{+}$currents associated with the cloned $\mathrm{Na}^{+} / \mathrm{I}^{-}$symporter (Eskandari et al. 1997). All experiments were performed with oocytes 4-6 days after injection. The oocytes were clamped to a potential of $-40 \mathrm{mV}$ and the current required for holding this potential was recorded with a Dagan TEV-200A Two Electrode Voltage Clamp.

During each assay, single cells were superfused constantly (at a flow rate of about $1.5 \mathrm{ml} / \mathrm{min}$ ) with an experimental medium (TpNa (in $\mathrm{mM}$ ): $100 \mathrm{NaCl}, 2 \mathrm{KCl}, 1 \mathrm{MgCl}_{2}, 1$ $\mathrm{CaCl}_{2}$ and $10 \mathrm{~N}$-2-hydroxyethylpiperazine- $\mathrm{N}^{\prime}$-ethanesulphonic acid (HEPES) ( $\mathrm{pH} 7.5$ ) supplemented with iodide and/or ITB in the following order: 1) $50 \mu \mathrm{M} \mathrm{NaI}$, 2) $50 \mu \mathrm{M}$ ITB, 3) $50 \mu \mathrm{M} \mathrm{NaI} / 50 \mu \mathrm{M} \mathrm{ITB} / 0 \cdot 5 \%$ DMSO and 4) $50 \mu \mathrm{M}$ NaI. Perfusion with each of the iodide- and/or ITBcontaining solutions lasted for $50 \mathrm{~s}$ and was followed by perfusion with $\mathrm{TpNa}$ until washout of the iodide or the compound and return of the holding current to baseline was obtained. For IC50 determination, different concentrations of ITB (in $\mu \mathrm{M}: 5 ; 10 ; 20 ; 30 ; 40 ; 80$ ) were used. Each oocyte was discarded after step 4 . The compound's stock solutions were stored in DMSO at $20^{\circ} \mathrm{C}$, thawed and diluted 200-fold in $\mathrm{TpNa}$ immediately before use. All results shown are the average of at least three independent experiments.

\section{Statistical analysis}

S.E.M. was calculated using Microsoft Excel software for all experiments. Student's $t$-tests were also performed using SigmaStat (Systat Software Inc., London, UK).

\section{Results}

ITBs identified by high-throughput screening for their capacities to inhibit iodide uptake in NIS-expressing mammalian cells, have different effects on iodide-induced currents of NIS-expressing oocytes from $\mathrm{X}$. laevis

For each assay, we analyzed one ITB using one oocyte. The oocytes were clamped to a holding potential of $-40 \mathrm{mV}$. Each assay was carried out as follows. We first verified the activity of expressed NIS with an iodide-containing perfusion medium $(\mathrm{TpNa} / \mathrm{I})$. The addition of $50 \mu \mathrm{M} \mathrm{I}^{-}$to the perfusion medium caused an inward current of $~ 30 \mathrm{nA}$. After washout of the iodide with $\mathrm{TpNa}$ the induced current rapidly returned to baseline as shown in Fig. 1A. We then investigated the effect of the ITB alone on the holding current (perfusion with medium $\mathrm{TpNa} / \mathrm{ITB}$ ). Most often the compounds by themselves showed no significant effect on the oocyte membrane conductance (e.g. see ITB 5 in
Fig. 1A). Only in some cases, an effect of the compound itself was observed (e.g. see ITB-4 in Fig. 1A). In general, these effects were reversible and disappeared upon withdrawal of the ITB so that the current rapidly returned to baseline. During the following step the ability of the ITB to abolish the iodide-evoked inward current was tested, and the holding potential was measured in the presence of both, iodide and the ITB (perfusion with $\mathrm{TpNa} / \mathrm{I} / \mathrm{ITB}$ ). If the ITB has a direct inhibitory effect on the NIS-catalyzed symport activity, the presence of iodide in the perfusion medium should no longer lead to the appearance of the iodide-dependent NIScharacteristic inward current. This is illustrated with ITB-5 in Fig. 1A. During the last step of our standard procedure inhibition reversibility was studied. The oocyte was perfused with $\mathrm{TpNa}$ until baseline current was re-established, and then again iodide was added to the perfusion medium. All compounds were analyzed following the described standard procedure with NIS- and water-injected oocytes.

The effects of ITB-1-ITB-10 are illustrated in Figs 1 and 2. The compounds were listed according to their efficacy to rapidly abolish iodide-evoked inward currents. Figure 1 shows the results obtained for a first group of four compounds (for chemical structures see Fig. 1D) that either completely inhibited the iodide current (see ITB-3) or showed at least an inhibition of more than $90 \%$ (see ITB-9, ITB-5 and ITB-4). Figure $1 \mathrm{~A}$ illustrates typical current recordings obtained with ITB-3, ITB-9, ITB-5, ITB-4 and DMSO (the solvent used for solubilization of all compounds). DMSO by itself did not alter the oocyte membrane conductance and had no effect on the iodide-induced inward current. ITB-3, ITB-9 and ITB-5 abolished more than $95 \%$ of the iodide current (Fig. 1B). When these compounds were washed out by perfusion with $\mathrm{TpNa}$ for several minutes, only $0-18 \%$ of the initial iodideinduced current was re-established (Fig. 1C). However, the inhibitory effect of ITB-4 $(95 \pm 8 \%)$ was reversible and $89 \cdot 5 \pm 9 \cdot 7 \%$ of the initial iodide current was restored after washout. We also found that ITB-4 by itself, led to the apparition of an outward current of $9 \pm 5 \cdot 6 \mathrm{nA}$ (see recording Fig. 1A). This phenomenon, i.e. the alteration of the oocyte membrane conductance induced by a compound itself will be discussed below.

Figure 2 illustrates the results obtained with the ITB-6, ITB-8, ITB-7, ITB-2, ITB-1 and ITB-10. The chemical structures of these molecules are shown in Fig. 2C. The inhibitory effect of these compounds on the iodide current was less than $80 \%$ (Fig. 2A). Briefly, ITB-6, ITB-7 and ITB-8 abolished about $72 \%$ of the iodide current (Fig. 2A) and the washout of the compounds led to the recovery of $67 \%$ of the initial iodide current (Fig. 2B). ITB-1 and ITB-2 caused a blocking effect of roughly $50 \%$ of the iodide-induced current, and this inhibitory effect persisted after washout of the compounds and addition of $50 \mu \mathrm{M}$ iodide (Fig. 2B). ITB-10 only slightly impaired the iodide-induced current (Fig. 2A). However, this small inhibitory effect was not reversible (Fig. 2B). More precisely, the ITB-6, ITB-8, ITB-7, ITB-2, ITB-1 and ITB-10 showed inhibitions of the iodide current 
A
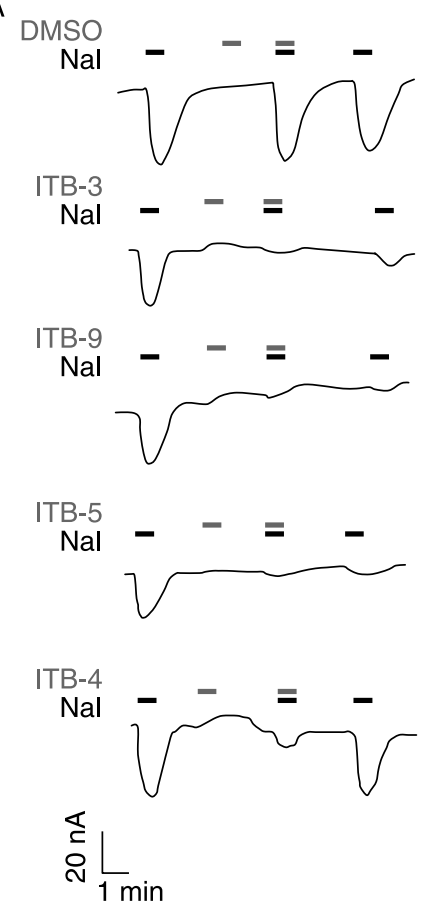

B

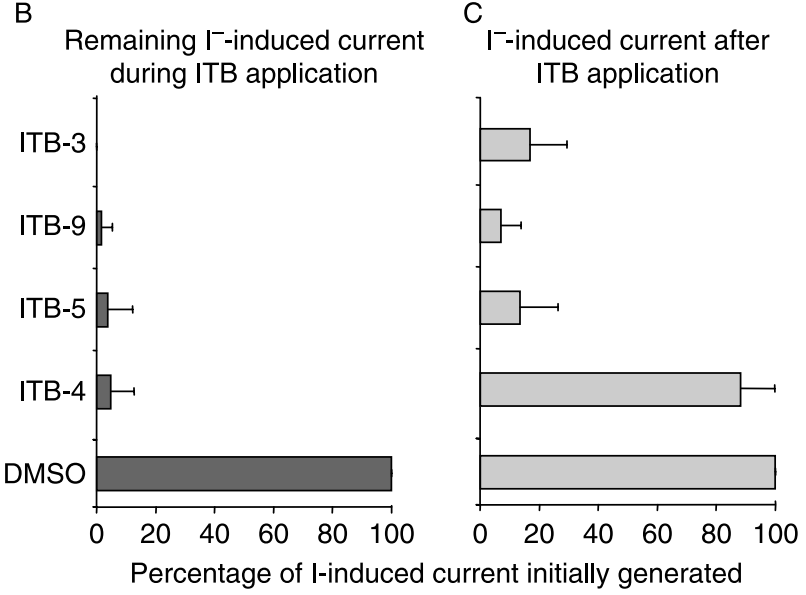

D

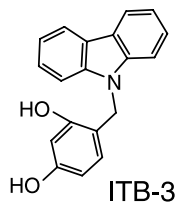

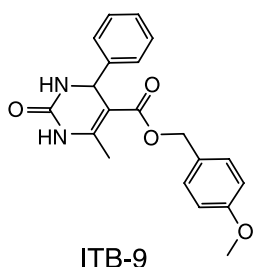

ITB-9

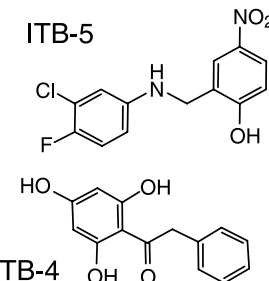

Figure 1 Effects of ITB-3, ITB-9, ITB-5 and ITB-4 on iodide-induced currents of NIS-expressing oocytes from Xenopus laevis. (A) Representative current recordings from voltage clamped ( $-40 \mathrm{mV}$ ) mNIS cRNA-injected oocytes superfused with ITB-3, ITB-9, ITB-5, ITB-4, DMSO and/or Nal following our standard protocol indicated in the top panel: $50 \mu \mathrm{M} \mathrm{Nal}$ (black bars); $50 \mu \mathrm{M}$ ITB/0.5\% DMSO (grey bars). Baseline was established in buffer TpNa. (B) Inhibition of iodide-induced currents. Inward currents induced by $50 \mu \mathrm{M}$ Nal in the presence of $50 \mu \mathrm{M} \mathrm{ITB} / 0 \cdot 5 \%$ DMSO were expressed as a percentage of the initial current generated by $50 \mu \mathrm{M} \mathrm{Nal}$ with the same oocytes. Currents were normalized with respect to the iodide current generated in the presence of $0 \cdot 5 \%$ DMSO, the solvent used for all compounds (full bars). (C) Reversibility of the inhibitory effect. Oocytes were superfused with $50 \mu \mathrm{M}$ Nal after drug application and its washout, i.e. return of the current to baseline. The generated currents are expressed as percentage of the initially obtained iodide current and are normalized with respect to the current generated by iodide after DMSO application (empty bars). Data are reported as mean \pm S.E.M. from at least three independent experiments. (C) Chemical structure of ITB3, ITB-9, ITB-5 and ITB-4.

of $76 \cdot 8 \pm 5,72 \pm 10,69 \pm 7,48 \pm 14,44 \pm 7$ and $21 \pm 8 \%$ respectively. After removal of the compounds and addition of $50 \mu \mathrm{M}$ iodide $65 \pm 8 \cdot 5,62 \pm 11,74 \pm 9,57 \pm 5,41 \pm 2 \cdot 5$ and $76 \pm 5 \%$ respectively of the initially observed iodide currents were restored.

To assure that the concentration of $50 \mu \mathrm{M}$ results in a maximal blocking effect we determined the IC50 values for the four most effective molecules. We found $5.87 \mu \mathrm{M} \pm$ $0 \cdot 87 \mu \mathrm{M}$ for ITB-9, $4.18 \mu \mathrm{M} \pm 1 \cdot 1 \mu \mathrm{M}$ for ITB-3, $7 \cdot 9 \mu \mathrm{M} \pm 2 \cdot 4 \mu \mathrm{M}$ for ITB-5 and $12 \mu \mathrm{M} \pm 1.9 \mu \mathrm{M}$.

Other molecules, described as inhibitors of the iodide uptake in NIS-expressing mammalian cells, have small effects on iodideinduced currents in NIS-expressing X. laevis oocytes

We also analyzed the effect of the organic molecules dysidenin, isodysidenin, ouabain and DIDS on iodideinduced currents in X. laevis oocytes (Fig. 3). Dysidenin, a toxin from the marine sponge $D$. herbacea (for the chemical structure see Fig. 3C) has been described as a specific inhibitor of the $\mathrm{Na}^{+} / \mathrm{I}^{-}$symporter in bovine thyroid slices, bovine plasma membrane vesicles and in NIS-expressing mammalian cell lines by Vroye et al. (1998). In our experiments, synthesized dysidenin showed an inhibition of $42 \pm 21 \%$ of the iodide-induced current (Fig. 3A). The inhibitory effect of dysidenin was fully reversible (Fig. 3B).

With isodysidenin, a synthesized epimer of dysidenin (Fig. 3C) we observed an inhibition of $29 \pm 5 \%$ of the iodideinduced inward current (Fig. 3A). However, in contrast to dysidenin, the inhibitory effect of isodysidenin was irreversible (Fig. 3B).

We also tested (as negative controls), ouabain and 4,4'di-isothiocyano-2, $2^{\prime}$-stilbene disulphonate (DIDS), that have been reported to affect iodide uptake in thyroid-derived cell lines or NIS-expressing cells but not via direct interaction with NIS. Ouabain, a well-known inhibitor of the $\mathrm{Na}^{+} / \mathrm{K}^{+}$ATPase leads to a decrease of the transmembrane sodium gradient, and thus affects the energy supply necessary for 


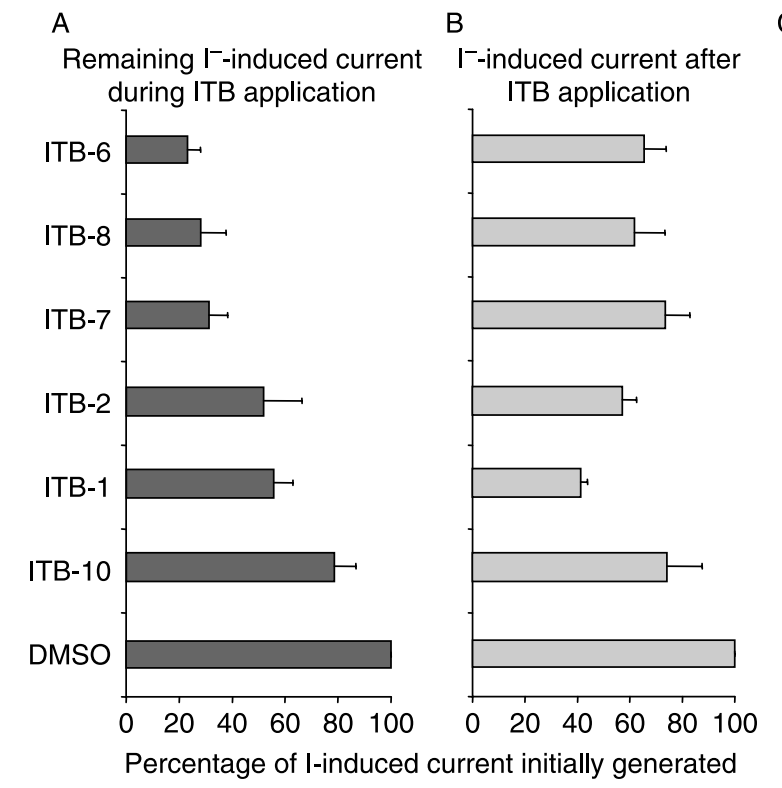

C
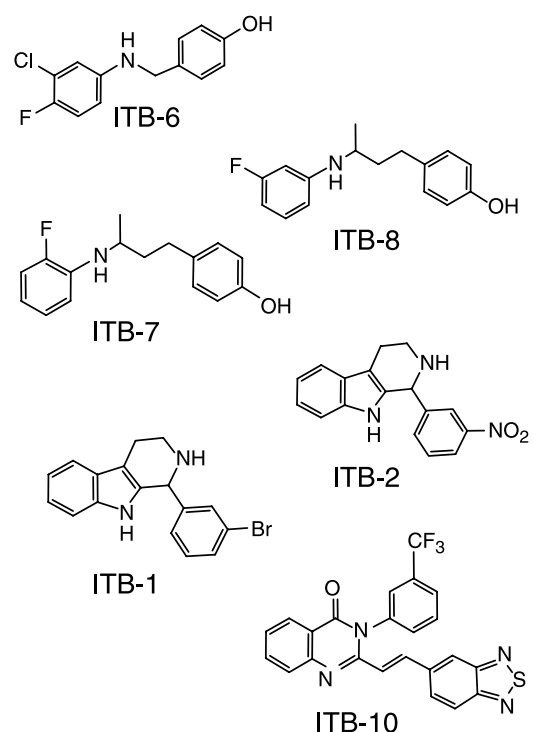

Figure 2 Effects of ITB-6, ITB-8, ITB-7, ITB-2, ITB-1 and ITB-10 on iodide-induced currents of NIS-expressing oocytes from Xenopus laevis. (A) Inhibition of iodide-induced currents in the presence of ITB-6, ITB-8, ITB-7, ITB-2, ITB-1, ITB-10 and DMSO. The membrane potential was clamped at $-40 \mathrm{mV}$. Currents were recorded from mNIS cRNA-injected oocytes following our standard protocol. Inward currents induced by $50 \mu \mathrm{M} \mathrm{Nal}$ in the presence of $50 \mu \mathrm{M} \mathrm{ITB} / 0 \cdot 5 \%$ DMSO were expressed as a percentage of the initial current obtained with $50 \mu \mathrm{M} \mathrm{Nal}$ with the same oocytes. Currents were normalized with respect to the iodide current generated in the presence of $0.5 \%$ DMSO, the solvent used for all compounds (full bars). (B) Reversibility of the inhibitory effect. Oocytes were superfused with $50 \mu \mathrm{M} \mathrm{Nal}$ after ITB application and its washout, i.e. return of the current to baseline. Baseline was established in buffer TpNa. The generated currents are expressed as percentage of the initially obtained iodide current and are normalized with respect to the current generated by iodide after DMSO application $(0 \cdot 5 \%$, empty bars). Data are reported as mean \pm s.e. from at least three independent experiments. (B) Chemical structure of ITB-6, ITB-8, ITB-7, ITB-2, ITB-1, ITB-10.

iodide transport (Tyler et al. 1968). However, ouabain does not directly interact with NIS and should not show a rapid effect on the iodide current in oocytes measured by voltage clamp. As expected, no inhibition of the iodide-induced current was observed with this compound (Fig. 3A). DIDS, a well-known chloride channel blocker, was reported to induce an increase of the radioactive iodide uptake in NIS-expressing cells by Chow et al. (1987) and Amphoux-Fazekas et al. (1998). The results obtained by both groups indicate that this effect is due rather to an inhibition of the iodide efflux than to a direct activation of NIS activity. As expected, we did not observe an inhibition of the iodide-induced current with DIDS (Fig. 3A).

ITBs have different effects on the basal membrane conductance of NIS-expressing or water-injected oocytes

Figure 4 shows the effects of the different compounds on the membrane conductance of NIS-expressing oocytes in the absence of iodide. ITB-3, ITB-9, ITB-4 and dysidenin evoked significant outward currents of $4 \pm 2 \cdot 4,9 \pm 3 \cdot 2,7 \cdot 5 \pm$ $5 \cdot 4$ and $8 \pm 3 \cdot 5 \mathrm{nA}$ respectively. The amplitude of these small currents varied widely as shown in Fig. 4. With the exception of dysidenin, these compounds also led to almost full inhibition of the iodide currents shown in Fig. 1. In contrast to dysidenin, no current was found with isodysidenin. As expected, ouabain induced an inward current $(8 \cdot 25 \pm$ $0.35 \mathrm{nA}$ ) that was due to the inhibition of the sodium efflux catalyzed by the $\mathrm{Na}^{+} / \mathrm{K}^{+}$-ATPase. No current was induced in the presence of DIDS.

In order to examine whether the above described effects of the compounds on the oocyte membrane conductance in the absence of iodide required NIS expression, we tested all the ITBs with water-injected oocytes. Only ITB- 5 evoked by itself a significant change in the membrane conductance in control oocytes (data not shown). ITB-5 evoked inward currents of $4 \cdot 1 \pm 1 \cdot 15 \mathrm{nA}$ but did not induce a significant current in NIS-expressing oocytes.

\section{Discussion}

In this work, we report the characterization of potential NISspecific inhibitors using the $X$. laevis oocyte expression system and voltage clamp. The 10 selected compounds were previously tested with NIS-expressing mammalian cells. They all showed efficient inhibition of radioactive iodide uptake in HEK-293 cells stably transfected with hNIS and in 


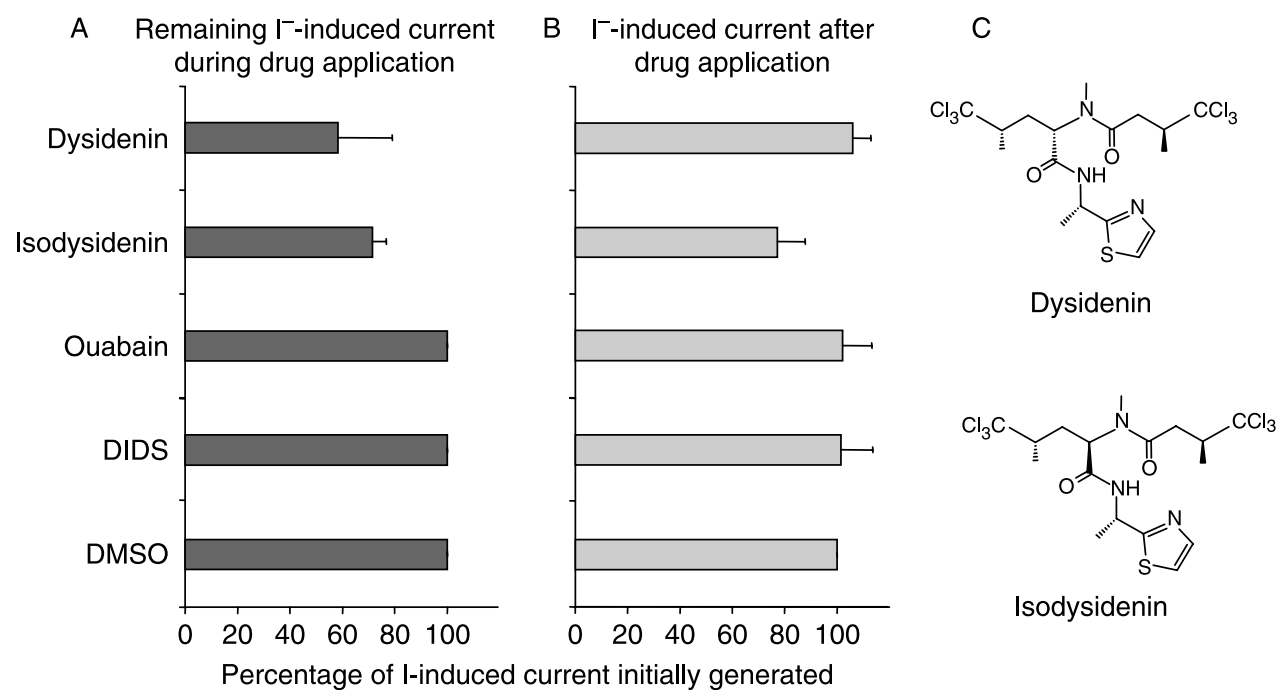

Figure 3 Effects of dysidenin, isodysidenin, ouabain and DIDS on iodide-induced currents of NIS-expressing oocytes from Xenopus laevis. (A) Inhibition of iodide-induced currents in the presence of dysidenin, isodysidenin, ouabain, DIDS and DMSO. The membrane potential was clamped at $-40 \mathrm{mV}$. Currents were recorded from mNIS cRNA-injected oocytes following our standard protocol. Inward currents induced by $50 \mu \mathrm{M} \mathrm{Nal}$ in the presence of $50 \mu \mathrm{M}$ compound/0.5\% DMSO were expressed as a percentage of the initial current obtained with $50 \mu \mathrm{M} \mathrm{Nal}$ with the same oocytes. Currents were normalized with respect to the iodide current generated in the presence of $0.5 \%$ DMSO, the solvent used for all compounds (full bars). (B) Reversibility of the inhibitory effect. Oocytes were superfused with $50 \mu \mathrm{M} \mathrm{Nal}$ after compound application and its washout. Baseline was established in buffer TpNa. The generated currents are expressed as percentage of the initially obtained iodide current and are normalized with respect to the current generated by iodide after DMSO application $(0.5 \%$, empty bars). Data are reported as mean \pm S.E.M. from at least three independent experiments. (C) Chemical structure of dysidenin and isodysidenin.

rat thyroid-derived FRTL-5 cells. Consequently, they were named ITB for 'Iodide Transport Blocker' (Lecat-Guillet et al. 2008). The aim of the analysis presented in this paper was to further characterize these ITBs, i.e. to identify those with a specific and rapid inhibitory effect on NIS activity. In addition, the reversibility of the drug effect was assessed.

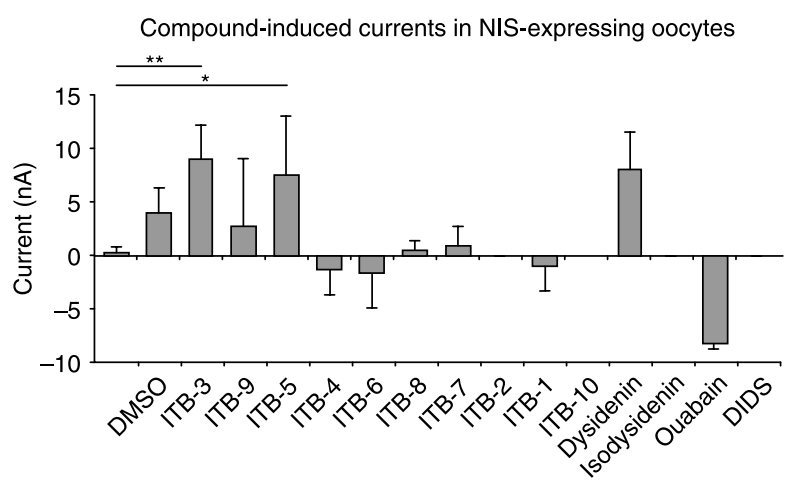

Figure 4 Effect of the compounds, by themselves, on the membrane conductance of NIS-expressing oocytes. The membrane potential was clamped at $-40 \mathrm{mV}$. Currents induced by all compounds $(50 \mu \mathrm{M}) / 0 \cdot 5 \%$ DMSO were recorded in the absence of iodide. Data are reported as mean \pm s.E.M. from at least three independent experiments. ${ }^{* *} P<0 \cdot 001 .{ }^{*} 0 \cdot 01<P<0 \cdot 02$.
The ITB's IC50 have been determined with cultured mammalian cell lines. They were found to be $0 \cdot 4-12 \mu \mathrm{M}$ for hNIS-expressing HEK-293 cells and 0.04-2 $\mu$ M for FRTL-5 cells. In order to simplify our analysis with $X$. laevis oocytes, all compounds were tested at a final concentration of $50 \mu \mathrm{M}$. We assumed that this concentration allowed for the highest inhibitory effects for all compounds. Nevertheless, we determined the IC50 values for the four more effective molecules, i.e. ITB-9, ITB-3, ITB-4 and ITB-5. The obtained values are somewhat higher than those reported by Lecat et al. with mammalian cells but a maximal inhibitory effect can be expected under our experimental conditions. For the same reason, iodide was used at a concentration of $50 \mu \mathrm{M}$ in our standard procedure. This iodide concentration is roughly thrice that of the mNIS transport constant $\left(K_{\mathrm{t}}=\right.$ $15 \mu \mathrm{M})$ that was previously determined with expressing oocytes (data not shown).

Our analysis revealed that some of the identified compounds do not rapidly and completely inhibit the NIS. Indeed, ITB-2 and ITB-1 inhibited less than 50\% of the iodide-induced current in NIS cRNA-injected oocytes. ITB-10 showed the smallest effect with only $21 \pm 8 \%$ inhibition. Interestingly, when the time-dependent inhibition of these compounds was studied on radioactive iodide uptakes on NIS-expressing mammalian cells a delayed inhibition was observed (Lecat-Guillet et al. 2008). This finding indicates 
that the effect of ITB-2, ITB-1 and ITB-10 on the iodide uptake may not be due to specific inhibition of NIS activity. One could suggest that these compounds act via the disruption of the transmembrane sodium gradient caused by an inhibition of the $\mathrm{Na}^{+} / \mathrm{K}^{+}$-ATPase activity. However, in voltage clamp experiments, the effect of ouabain (a wellknown $\mathrm{Na}^{+} / \mathrm{K}^{+}$-ATPase inhibitor) on the oocyte transmembrane conductance differed significantly from the effects obtained with ITB-2, ITB-1 and ITB-10. As expected, in NIS-expressing oocytes, ouabain, by itself, induced an inward current due to the inhibition of the $\mathrm{Na}^{+} / \mathrm{K}^{+}$pump and had no effect on the iodide-induced current. By contrast, ITB-2, ITB-10 and ITB-1, by themselves, evoked only small and non-significant currents on the membrane conductance in the absence of iodide. In contrast to ouabain, the three compounds showed an inhibitory effect on the iodide current that was not reversible. In addition, long-term incubation (more than $24 \mathrm{~h}$ ) of cultured mammalian cells with ITB-2, ITB-1 and ITB-10 did not affect cell viability (Lecat-Guillet et al. 2008) as would be expected for inhibitors of the $\mathrm{Na}^{+} / \mathrm{K}^{+}$pump. We therefore conclude that the delayed inhibition of ITB-2, ITB-1 and ITB-10 was not due to their effect on the transmembrane sodium gradient but rather to their effect on post-transcriptional regulatory pathways of the NIS protein. The observed inhibition of the iodide current could also require an interaction of these compounds with cytoplasmic portions of NIS and would then depend on their accumulation rate in the cytoplasm. In this case, the passage of the compounds through the plasma membrane would be the rate-limiting step. The drugs could also bind only slowly to the NIS protein and irreversibly inactivate the transport activity. Considering the results obtained by the high-throughput screening and by voltage clamp, we suggest that NIS activity is inhibited by ITB-2, ITB-1 and ITB-10 but inhibition occurs slowly requiring longer incubation periods (more than $1 \mathrm{~h}$ ) of the cells with the compound. A similar phenomenon, i.e. a delayed inhibitory effect was also reported for the $\mathrm{Na}^{+} / \mathrm{H}^{+}$-exchanger isoform NHE3 by squalamine (Akhter et al. 1999) but unfortunately, the molecular mechanism of this process also remains unknown. Based on our results, we suggest that ITB-2, ITB-1 and ITB-10 mediate mainly irreversible and progressive inactivation of NIS.

Perfusion of the injected oocytes with ITB-6, ITB-8 and ITB-7 led to a rapid though not complete inhibition of the iodide-induced current; roughly $25 \%$ of the initially measured current remained in the presence of the compounds. This remaining current is difficult to explain since a delayed inhibition on radioactive iodide uptakes on NIS-expressing mammalian cells for these ITBs was not observed (Lecat-Guillet et al. 2008) and they were used on $X$. laevis oocytes at a concentration exceeding several times their IC50 (13, 14.7 and $14.3 \mu \mathrm{M}$ respectively; data not shown). In addition, only non-significant currents were induced by ITB-6, ITB-8 and ITB-7 by themselves, suggesting a NIS-specific action. However, the inhibitory effect of these molecules was mainly reversible indicating that a progressive inactivation of the NIS did not occur.

The purified toxin, dysidenin was reported by Vroye et al. (1998) to be a specific and potent NIS inhibitor. The authors found a $K_{\mathrm{i}}$ of $5 \mu \mathrm{M}$ measuring the initial iodide uptake rates in thyroid plasma membrane vesicles in the presence of the toxin. More than $90 \%$ of the iodide uptake was restored upon removal of the toxin. By contrast, prolonged incubation (120 min) with $100 \mu \mathrm{M}$ dysidenin was necessary to obtain about 50\% inhibition with hNIS-expressing HEK-293 cells (Lecat-Guillet et al. 2008). Inhibition was complete with FRTL-5 cells after an incubation period of $60 \mathrm{~min}$. An IC50 value for dysidenin of $\sim 2 \mu \mathrm{M}$ with hNIS-expressing HEK293 cells and of $\sim 6 \mu \mathrm{M}$ with FRTL-5 cells was found. We report in this work that dysidenin $(50 \mu \mathrm{M})$ led to a rapid and reversible inhibition of about $40 \%$ of the iodide-induced current in mNIS-expressing oocytes. The inhibition varied significantly between oocytes from the same batch (see standard error in Fig. 3), which may be due to the poor aqueous solubility of the toxin. Dysidenin can be fully dissolved in DMSO, but a precipitate appears after its dilution with perfusion medium to working concentration. Therefore, it is difficult to conclude that the whole effect of dysidenin on the iodide-induced current in oocytes is directly associated with NIS inhibition. Isodysidenin, the epimer of dysidenin, led to a rapid and irreversible inhibition of only $15 \%$ of the iodide-induced current. Both compounds did not evoke currents in the absence of iodide suggesting a specific effect on NIS activity. This finding is not in agreement with the inhibitions reported for isodysidenin by Vroye et al. (1998). The authors used dysidenin and isodysidenin purified from the dichloromethane extract of the marine sponge $D$. herbacea. The compounds we used were obtained from a mixture kindly provided by Prof. J.C. Braekman and were further purified with HPLC. We cannot exclude differences in the purity of the compounds. Small impurities in the compound preparations may account for the discrepancies described above.

Four of the identified compounds provoked a rapid inhibition of NIS: ITB-3, ITB-9, ITB-5 and ITB-4. These compounds blocked more than $90 \%$ of the iodide-induced current but the mechanism of the inhibition differed markedly between the compounds. The inhibitory effects of ITB-3, ITB-9 and ITB-5 were not reversible suggesting a low $K_{\mathrm{d}}$ for the association ITB/NIS. In this case, dissociation of the compound from NIS would be a very slow process and should require longer perfusion periods than those used in our standard protocol. The compounds could also lead to an irreversible modification of the NIS protein conformation abolishing its transport activity. However, further studies on the kinetics of the compound-induced inhibition of the iodide current at lower concentrations and of the currents during washout should allow a better understanding of the molecular mechanisms involved.

In addition, we observed that ITB-3 and ITB-9 by themselves induced significant currents with NIS-expressing oocytes. Similar, though statistically not significant effects 
were also found with control oocytes indicating that these compounds were modifying the activity of transporters and/or channels endogenously expressed in the oocytes. Such outward currents could result from an increase of the potassium efflux, the inhibition of the sodium influx and/or the chloride efflux. As no toxic effects of these compounds with HEK-293 and FRTL-5 cells were observed (Lecat-Guillet et al. 2008), we are confident that the effects of ITB-3 and ITB-9 on endogenous mammalian proteins will prove to be insignificant allowing their future use for clinical applications. ITB-5 induced an inward current with waterinjected oocytes. This effect was not found in NIS-expressing oocytes in the absence of iodide. Thus, in addition to its effect on presumed endogenous proteins in water-injected oocytes the compound may also have a NIS-specific effect.

In contrast to ITB-3, ITB-9 and ITB-5, the strong inhibitory effect of ITB-4 on the iodide-induced current was fully reversible, i.e. the initially measured iodide current with the same oocytes was completely restored after washout of the compound. This behaviour is expected from a molecule that reversibly binds to the transporter reducing substrate recognition or translocation. ITB-4 also induced an outward current in NIS-expressing oocytes in the absence of iodide which was not observed in control oocytes. Similar outward currents were also observed with ITB-9 and ITB-3. This allows speculating on the mechanisms involved in NIS inhibition. One of the simplest hypotheses, we suggest is that the binding of the compound to the NIS protein alters the sodium recognition site that, in turn, provokes a decrease of the sodium-dependent inward leak current (Eskandari et al. 1997). Alternatively, ITB-4, ITB-9 and ITB-3 could inhibit a translocation step required for the sodium-coupled iodide transport and the sodium-dependent leak.

In conclusion, the most promising identified compounds are ITB-3, ITB-9, ITB-5 and ITB-4. In contrast to perchlorate, these compounds are non-competitive NIS inhibitors. They certainly represent efficient tools for further functional studies of NIS and should also prove helpful elucidating NIS regulation. However, for clinical use, the solvent DMSO cannot be used. Further studies of the physical and chemical behaviour of the molecules (or derived compounds) in aqueous solutions and of a suitable administration procedure are required. If no major side effect is associated with the administration of the compounds, several medical applications for NIS-specific inhibitors can be proposed. i) In cases of treatment of hyperthyroidism that frequently occurs during administration of amiodarone, the identified compounds could be a useful option to the commonly used drugs. The effects of these drugs are essentially based on their ability to inhibit iodide organification which often causes various undesirable side effects. ii) In the diagnosis of thyroid pathology, perchlorate is used to study the thyroid capacity to incorporate iodide. Specific inhibitors of NIS could constitute a less toxic drug. iii) In the case of radioiodine contamination, the administration of a NISspecific inhibitor would prevent an accumulation of the isotope in the thyroid, and thus, lead to an important reduction of the thyroid radiation dose. While blocking the entrance of radioactive iodide, the treatment will still allow the clearing of the thyroid from unbound isotope and the secretion of radioactive iodine-containing hormone precursors (Dayem et al. 2006). (iv) Finally, targeted NIS gene transfer followed by radioiodine therapy is a very promising strategy in cancer treatment. The transfer of an inhibitorresistant NIS gene into cancer cells and the use of a specific NIS inhibitor during radiotherapy should prevent the accumulation of radioactive iodide in non-malignant cells endogenously expressing NIS (i.e. cells from thyroid, salivary gland, stomach, etc.).

\section{Declaration of interest}

There are no conflicts of interest.

\section{Funding}

This work was supported by grants from EDF. A O-M was funded by ECOSNord.

\section{Acknowledgements}

The authors wish to thank Dr Patrick Chang for critical reading of the manuscript.

\section{References}

Akhter S, Nath SK, Tse CM, Williams J, Zasloff M \& Donowitz M 1999 Squalamine, a novel cationic steroid, specifically inhibits the brush-border $\mathrm{Na}^{+} / \mathrm{H}^{+}$exchanger isoform NHE3. American Journal of Physiology 276 C136-C144

Amphoux-Fazekas T, Samih N, Hovsepian S, Aouani A, Beauwens R \& Fayet G 1998 DIDS ( $4^{\prime}$-diisothiocyanatostilbene-2,2' -disulfonic acid) increases iodide trapping, inhibits thyroperoxidase and antagonizes the TSHinduced apical iodide efflux in porcine thyroid cells. Molecular and Cellular Endocrinology 141 129-140.

Bartalena L, Wiersinga WM, Tanda ML, Bogazzi F, Piantanida E, Lai A \& Martino E 2004 Diagnosis and management of amiodarone-induced thyrotoxicosis in Europe: results of an international survey among members of the European Thyroid Association. Clinical Endocrinology 61 494-502.

Cho JY, Leveille R, Kao R, Rousset B, Parlow AF, Burak WE Jr, Mazzaferri EL \& Jhiang SM 2000 Hormonal regulation of radioiodide uptake activity and $\mathrm{Na}^{+} / \mathrm{I}^{-}$symporter expression in mammary glands. Journal of Clinical Endocrinology and Metabolism 85 2936-2943.

Chow SY, Yen-Chow YC, White HS \& Woodbury DM 1987 Effects of 4,4' di-isothiocyano-2, $2^{\prime}$-stilbene disulphonate on iodide uptake by primary cultures of turtle thyroid follicular cells. Journal of Endocrinology 113 403-412.

Clewell RA, Merrill EA, Narayanan L, Gearhart JM \& Robinson PJ 2004 Evidence for competitive inhibition of iodide uptake by perchlorate and translocation of perchlorate into the thyroid. International Journal of Toxicology 23 17-23.

Dadachova E, Bouzahzah B, Zuckier LS \& Pestell RG 2002 Rhenium-188 as an alternative to Iodine-131 for treatment of breast tumors expressing the sodium/iodide symporter (NIS). Nuclear Medicine and Biology 29 13-18.

Dai G, Levy O \& Carrasco N 1996 Cloning and characterization of the thyroid iodide transporter. Nature 379 458-460. 
Dayem M, Navarro V, Marsault R, Darcourt J, Lindenthal S \& Pourcher T 2006 From the molecular characterization of iodide transporters to the prevention of radioactive iodide exposure. Biochimie 88 1793-1806.

Dohan O \& Carrasco N 2003 Advances in $\mathrm{Na}(+) / \mathrm{I}(-)$ symporter (NIS) research in the thyroid and beyond. Molecular and Cellular Endocrinology 213 $59-70$.

Dohan O, De La Vieja A, Paroder V, Riedel C, Artani M, Reed M \& Ginter CS 2003 The sodium/iodide symporter (NIS): characterization, regulation, and medical significance. Endocrine Reviews 24 48-77.

Dohan O, Portulano C, Basquin C, Reyna-Neyra A, Amzel LM \& Carrasco N $2007 \mathrm{The} \mathrm{Na}^{+} / \mathrm{I}^{-}$symporter (NIS) mediates electroneutral active transport of the environmental pollutant perchlorate. PNAS 104 20250-20255.

Eskandari S, Loo DDF, Dai G, Levy O, Wright EM \& Carrasco N 1997 Thyroid $\mathrm{Na}^{+} / \mathrm{I}^{-}$symporter. Mechanism, stoichiometry, and specificity. Journal of Biological Chemistry 272 27230-27238.

Gerard C, Rigot V \& Penel C 1994 Chloride channel blockers inhibit the $\mathrm{Na}^{+} / \mathrm{I}^{-}$symporter in thyroid follicles in culture. Biochemical and Biophysical Research Communications 204 1265-1271.

Kaminsky SM, Levy O, Garry MT \& Carrasco N 1991 Inhibition of the $\mathrm{Na}^{+} / \mathrm{I}^{-}$symporter by harmaline and 3-amino-1-methyl-5H- pyrido(4,3b)indole acetate in thyroid cells and membrane vesicles. European Journal of Biochemistry 200 203-207.

Lecat-Guillet N, Merer G, Lopez R, Pourcher T, Rousseau B \& Ambroise Y 2007 A 96-well automated radioiodide uptake assay for sodium/iodide symporter inhibitors. Assay and Drug Development Technologies 5 535-540.

Lecat-Guillet N, Merer G, Lopez R, Pourcher T, Rousseau B \& Ambroise Y 2008 Small-molecule inhibitors of sodium iodide symporter function. Chembiochem 9 889-895.

Lindencrona U, Nilsson M \& Forssell-Aronsson E 2001 Similarities and differences between free 211At and 125I- transport in porcine thyroid epithelial cells cultured in bicameral chambers. Nuclear Medicine and Biology 28 41-50.
Perron B, Rodriguez AM, Leblanc G \& Pourcher T 2001 Cloning of the mouse sodium iodide symporter (mNIS) and its expression in the mammary gland and other tissues,. Journal of Endocrinology 170 185-196.

Van Sande J, Deneubourg F, Beauwens R, Braekman JC, Daloze D \& Dumont JE 1990 Inhibition of iodide transport in thyroid cells by dysidenin, a marine toxin, and some of its analogs. Molecular Pharmacology 37 583-589.

Van Sande J, Massart C, Beauwens R, Schoutens A, Costagliola S, Dumont JE \& Wolff J 2003 Anion selectivity by the sodium iodide symporter. Endocrinology 144 247-252.

Smanik PA, Liu Q, Furminger TL, Ryu K, Xing S, Mazzaferri EL \& Jhiang SM 1996 Cloning of the human sodium lodide symporter. Biochemical and Biophysical Research Communications 226 339-345.

Tazebay UH, Wapnir IL, Levy O, Dohan O, Zuckier LS, Hua Zhao Q, Fu Deng H, Amenta PS, Fineberg S, Pestell RG et al. 2000 The mammary gland iodide transporter is expressed during lactation and in breast cancer Nature Medicine 6 871-878.

Tyler DD, Gonze J, Lamy F \& Dumont JE 1968 Influence of mitochondrial inhibitors on the respiration and energy-dependent uptake of iodide by thyroid slices. Biochemical Journal 106 123-133.

Vroye L, Beauwens R, Van Sande J, Daloze D, Braekman JC \& Golstein PE $1998 \mathrm{The} \mathrm{Na}^{+}-\mathrm{I}^{-}$cotransporter of the thyroid: characterisation of new inhibitors. Pflügers Archiv 435 259-266.

Received in final form 6 November 2008

Accepted 3 December 2008

Made available online as an Accepted Preprint

3 December 2008 